\title{
2,6-Dithienyl-4-arylpyridines: Synthesis, Topoisomerase I and II Inhibition and Structure-activity Relationship
}

\author{
Pritam Thapa, Radha Karki, Arjun Basnet, Uttam Thapa, Hoyoung Choi, Younghwa Na, Yurngdong Jahng, \\ Chong-Soon Lee, ${ }^{\ddagger}$ Youngjoo Kwon, ${ }^{*}$ Byeong-Seon Jeong, and Eung-Seok Lee

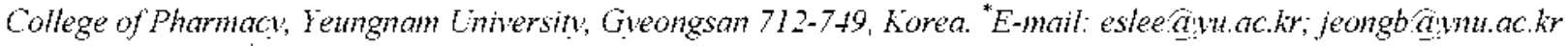 \\ ${ }^{\dagger}$ College of Phamacy Catholic Lniversity of Daegu, Gleongsan 712-702, Korea \\ -Department of Biochemistry, leungham Liniversitw, Gieongsan 712-749, Korea \\ ${ }^{\$}$ College of Pharmacy, Ewha Womans Lniversity, Seoul 120-750, Korea \\ Received June 13,2008
}

Key Words : 2.6-Dithieny l-4-aryllpyridines. Topoisomerase I and II inhibitors. Antitumor agents

Topoisomerases I and II are nuclear enzymes that play crucial roles in DNA metabolism such as replication. transcription. recombination. repair. chromatin assembly: and chromosome segregation. ${ }^{1-4}$ Due to the crucial role of topoisomerases in the maintenance and replication of DNA during proliferation. cells become highly vulnerable when these functions are lost. Consequently. topo I and II have been attractive targets for design of antitumor agents."

It has been reported in our research group $\alpha$-terpyridine ${ }^{7.8}$ derivatives showed strong cytotoxicities against several human cancer cell lines and considerable topo I and II inhibitory activities. We also studied for topo I and II inhibitory activities of 2.6-dithienyl-4-furylpyridines. ${ }^{10}$ From the previous structure-activity relationship studies. we found 2.6-dithienyl-4-furylpy ridines exhibited strong topo I and II inhibitory activities as well as strong cytotoxicity against several human cancer cell lines. It would be very interesting to prepare 2.6-dithienyl-4-arylpyridines and evaluate topo I and II inhibitory activities of the prepared compounds. In addition. the comparison of activities between two moieties may provide valuable infonmation for the development of novel antitumor agents. In connection with previous studies. 2.6-dithienyl-4-arylpyridines were prepared and evaluated for their topo I and II inhibitory effects (Figure 1).

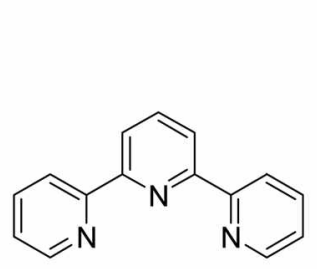

$\alpha$-terpyridine

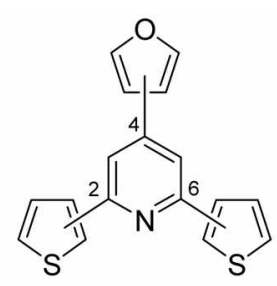

2,6-dithienyl-4-furylpyridines<smiles>[Al]c1cc(-c2ccsc2)nc(-c2cccs2)c1</smiles>

Aryl :
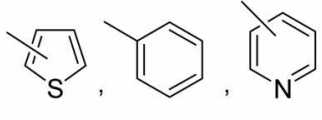

2,6-dithienyl-4-arylpyridines

Figure 1

\section{Experimental Section}

Compounds used as starting materials and reagents were purchased from Aldrich Chemical Co.. Sigma. Fluka. Junsei and used without further purification. Thin-layer chromatography (TLC) and columun chromatography were perfomed with Kieselgel $60 \mathrm{~F}_{254}$ (Merck) and silica gel (Kieselgel 60 . 230-400 mesh. Merck). respectively: Since all the compounds prepared contain aromatic rings. compounds were visualized and detected on TLC plates with UV light (short wave. long wave. or both). NMR spectra were recorded on a Bniker AMX $250\left(250 \mathrm{MHz}\right.$. FT) for ${ }^{1} \mathrm{H}$ NMR and 62.5 $\mathrm{MHz}$ for ${ }^{13} \mathrm{C}$ NMR and TMS (tetramethylsilane) was used as an intemal standard. Chemical shifts $(\delta)$ were recorded in ppm and coupling constants $(J)$ in hertz $(\mathrm{Hz})$. Melting points were determined in open capillary tubes on electrothennal 1A 9100 digital melting point apparatus and were uncorrected. ESI LC/MS analyses were performed with a Finnigan LCQ Advantage ${ }^{(\bar{B})}$ LC/MS/MS spectrometry utilizing $\mathrm{Xcalibur}^{\mathrm{E}}$ program. For ESI LC/MS. LC was performed with a $1 \mu \mathrm{L}$ injection volume on a Waters XTerra ${ }^{\mathbb{E}} 3.5 \mu \mathrm{m}$ reverse-phase $\mathrm{C}_{18}$ colunn $(2.1 \times 100 \mathrm{~mm})$ with a gradient elution from $5 \%$ to $95 \%$ of B in A for 20 min followed by $95 \% \mathrm{~B}$ in A for $10 \mathrm{~min}$ at a flow rate of $200 \mu \mathrm{L} / \mathrm{min}$. where mobile phase A was $100 \%$ distilled water with $50 \mathrm{mM}$ ammonium formate and mobile phase B was $100 \%$ acetonitrile. MS ionization conditions were: Sheath gas flow rate: 70 arb. aux gas flow rate: 20 arb. I spray voltage: $4.5 \mathrm{KV}$. capillary temp. $215^{\circ} \mathrm{C}$. capillary voltage: $21 \mathrm{~V}$. tube lens offset: $10 \mathrm{~V}$.

General method for the preparation of 3 . Aryl aldehyde 2 was added to the solution of $85 \% \mathrm{KOH}(1.2$ eq.) in $\mathrm{MeOH}$ $(50 \mathrm{~mL})$ and $\mathrm{H}_{2} \mathrm{O}(10 \mathrm{~mL})$ at $0^{\circ} \mathrm{C}$. After dissolution, thienyl methyl ketone (1. 1.0 eq.) was added over a period of 10 $\mathrm{min}$. The mixture was then stirred for $3 \mathrm{~h}$ at $0^{\circ} \mathrm{C}$. A solid product precipitated was filtered, washed with cold $\mathrm{MeOH}$ and dried to yield 47 to $99 \%$. Utilizing the same procedure. thirteen compounds were syinthesized.

1,3-Dithiophen-3-yl-propenone $\left\{3\left(R^{1}=b, R^{2}=b\right)\right\}$. A white solid ( $85 \%$ ). TLC (EtOAc $n$-hexane $=1: 2$ ) $\mathrm{R}_{\mathrm{i}}=0.5$. mp 77.1-78.2 ${ }^{\circ} \mathrm{C}$. ${ }^{1} \mathrm{H}$ NMR $\left(250 \mathrm{MHz} . \mathrm{CDCl}_{2}\right) \delta 8.14$ (dd. $J$ $=2.8 .1 .2 \mathrm{~Hz} .1 \mathrm{H} .1$-thiophen H-2). $7.80(\mathrm{~d} . J=15.5 \mathrm{~Hz} .1 \mathrm{H}$. 
$-\mathrm{CO}-\mathrm{C}=\mathrm{CH}-), 7.65(\mathrm{dd} . J=5.0 .1 .2 \mathrm{~Hz}, \mathrm{lH}$. 1-thiophen H5). $7.59(\mathrm{dd} . J=2.7,1.1 \mathrm{~Hz} .1 \mathrm{H}, 3$-thiophen $\mathrm{H}-2) .7 .4 \mathrm{l}(\mathrm{dd}, J$ $=5.0,1.1 \mathrm{~Hz}, \mathrm{LH} .3$-thiophen $\mathrm{H}-5$ ). 7.35 (dd, $J=5.0 .2 .8 \mathrm{~Hz}$. lH. 1-thiophen $\mathrm{H}-4$ ), 7.34 (dd. $J=5.0 .2 .7 \mathrm{~Hz}, 1 \mathrm{H}, 3-$ thiophen $\mathrm{H}-4) .7 .22(\mathrm{~d} . J=15.5 \mathrm{~Hz} .1 \mathrm{H} .-\mathrm{CO}-\mathrm{CH}=\mathrm{C}-)$.

General method for the preparation of 6-23. A mixture of $3\left(R^{1}=a-c . R^{2}=a-g\right)$, dry anmonium acetate and $\mathcal{4}\left(R^{3}=\right.$ a-b) in dry $\mathrm{MeOH}$ was heated at $80^{\circ} \mathrm{C}$ for 12 to $24 \mathrm{~h}$ under nitrogen atmosphere. The solvent was removed by evaporation under reduced pressure, and the residue was diluted with ethyl acetate $(100 \mathrm{~mL})$. washed with water $(75 \mathrm{~mL} \times 2)$ and saturated $\mathrm{NaCl}$ solution $(50 \mathrm{~mL}$ ). The organic layer was dried with magnesium sulfate and filtered. The filtrate was evaporated at reduced pressure. which was purified by silica gel column chromatography with a gradient elution of ethyl acetate/n-hexane to afford a white solid in 54 to $93 \%$ yields. Utilizing the same procedure, eighteen compounds were synthesized

4-(Thiophen-2-yl)-2,6-di(thiophen-3-yl)pyridine (10). A white solid $(54 \%)$, TLC (EtOAc/n-hexane $=1: 5)$. $\mathrm{R}_{\mathrm{t}}=0.4$. mp 155.9-156.5 ${ }^{\circ} \mathrm{C}$, ESI LC/MS/MS: Retention time: 6.84 mil, $[\mathrm{MH}]^{+}: 326.2 .{ }^{1} \mathrm{H} \mathrm{NMR}\left(250 \mathrm{MHz}, \mathrm{CDCl}_{3}\right) \delta 8.04$ (dd. $J=2.98,1.18 \mathrm{~Hz}, 2 \mathrm{H}$. 2-thiophene $\mathrm{H}-2,6$ "-thiophene $\mathrm{H}-2$ ). 7.79 (dd, $J=5.03,1.16 \mathrm{~Hz}, 2 \mathrm{H}, 2$-thiophene $\mathrm{H}-5,6$ "-thiophene $\mathrm{H}-5), 7.68$ (s, 2H. pyridine $\mathrm{H}-3, \mathrm{H}-5) .7 .58$ (dd, $J=$ $3.57,0.98 \mathrm{~Hz}, 1 \mathrm{H}, 4$ '-thiophene $\mathrm{H}-5$ ). 7.44 (dd. $J=5.01$. $0.99 \mathrm{~Hz}, 1 \mathrm{H}, 4$ 'thiophene $\mathrm{H}-3$ ), 7.42 (dd. $J=5.01 .3 .03 \mathrm{~Hz}$. 2H. 2-thiophene $\mathrm{H}-3,6$ "-thiophene $\mathrm{H}-3$ ), 7.17 (dd. $J=5.03$. $3.67 \mathrm{~Hz}, 1 \mathrm{H}, 4$ '-thiophene $\mathrm{H}-4),{ }^{13} \mathrm{C}$ NMR $(62.5 \mathrm{MHz}$. $\left.\mathrm{CDCl}_{3}\right) \delta 153.77 .142 .87 .142 .18,141.69,128.36 .126 .90$. 126.39. 126.17, 125.21. 123.92.114.76.

Pharmacology. The topoisomerase I inhibitory activity was carried out as followings: ${ }^{11}$ The activity of DNA topoisomerase I was determined by measuring the relaxation of supercoiled DNA pBR322. For measurement of topoisomerase I activity. the reaction mixture was comprised of $35 \mathrm{mM}$ Tris- $\mathrm{HCl}(\mathrm{pH} 8.0)$. $72 \mathrm{mM} \mathrm{KCl} 5 \mathrm{mM} \mathrm{MgCl} .5 \mathrm{mM}$ dithiothreitol. $2 \mathrm{mM}$ spermidine. $0.01 \%$ bovine senum albumin. $200 \mathrm{ng}$ pBR322. $0.3 \mathrm{U}$ calf thymus DNA topoisomerase I (Amersham) and topoisomerase I inhibitors (prepared compounds) in a final volume of $10 \mu \mathrm{L}$. The reaction mixture was incubated at $37^{\circ} \mathrm{C}$ for $30 \mathrm{~min}$. The reactions were terminated by adding $2.5 \mu \mathrm{L}$ of solution comprising $10 \%$ SDS. $0.2 \%$ bromophenol blue, $0.2 \%$ xylene cyanol and $30 \%$ glycerol. The mixture was applied to 1\% agarose gel and electrophoresed for $10 \mathrm{~h}$ with a ruming buffer of Trisborate-EDTA. Gels were stained for $30 \mathrm{~min}$ in a aqueous solution of ethidium bromide $(0.5 \mu \mathrm{g} / \mathrm{mL})$. DNA bands were visualized by transillumination with UV light and supercoiled DNA was quantitated by an image analyzer and LabWork 4.5 software (UVP).

The topoisomerase II inlibitory activity was carried out as followings: DNA topoisomerase II inlibition was measured by assessing relaxation of supercoiled pBR322 plasmid DNA. The reaction mixture contained $50 \mathrm{mM}$ Tris- $\mathrm{HCl}(\mathrm{pH}$ 8.0). $120 \mathrm{mM} \mathrm{KCl} .10 \mathrm{mM} \mathrm{MgCl}, 0.5 \mathrm{mM}$ ATP. $0.5 \mathrm{mM}$ dithiothreitol, $30 \mu \mathrm{g} / \mathrm{mL}$ bovine serum albumin, $0.2 \mu \mathrm{g}$ pBR322 plasmid DNA, $0.3 \mathrm{U}$ human DNA topoisomerase II $\alpha$ (TopoGEN), and topoisomerase II inhibitors (prepared compounds) in a final volume of $20 \mu \mathrm{L}$. The reactions were incubated for $30 \mathrm{~min}$ at $37^{\circ} \mathrm{C}$ and terminated by the addition of $3 \mu \mathrm{L}$ of solution containing $0.77 \%$ sodium dodecyl sulfate, $77 \mathrm{mM}$ EDTA. Samples were mixed with $2 \mu \mathrm{L}$ of solution containing of $30 \%$ sucrose, $0.5 \%$ bromophenol blue and $0.5 \%$ xylene cyanol, and subjected to electrophoresis on a $1 \%$ agarose gel at $1.5 \mathrm{~V} / \mathrm{cm}$ for $10 \mathrm{~h}$ with a running buffer of Tris-borate-EDTA. Gels were stained for $30 \mathrm{~min}$ in a aqueous solution of ethidium bromide $(0.5 \mu \mathrm{g} / \mathrm{mL})$. DNA bands were visualized by transillumination with UV light and supercoiled DNA was quantitated by an image analyzer and LabWork 4.5 software (UVP).

\section{Results and Discussion}

Chemistry. Synthetic methods for the preparation of 2.6dithienyl-4-aryl pyridines (6-23) are summarized in Scheme 1. Acetylthiophenes 1a-c were treated with arylcarboxaldelydes $\mathbf{2 a - g}$ in the presence of $\mathrm{KOH}$ in methanol-water (5:1), to afford internediates 3 in a $47-99 \%$ yields. Using modified Krōhnke ș̣nthesis. ${ }^{12}$ 2.6-dithienyl-4-arylpyridines were prepared by treatment of 3 with 1-(2-oxo-2-thienylethyl)pyridinium iodide (4a-b) in the presence of anmoni-
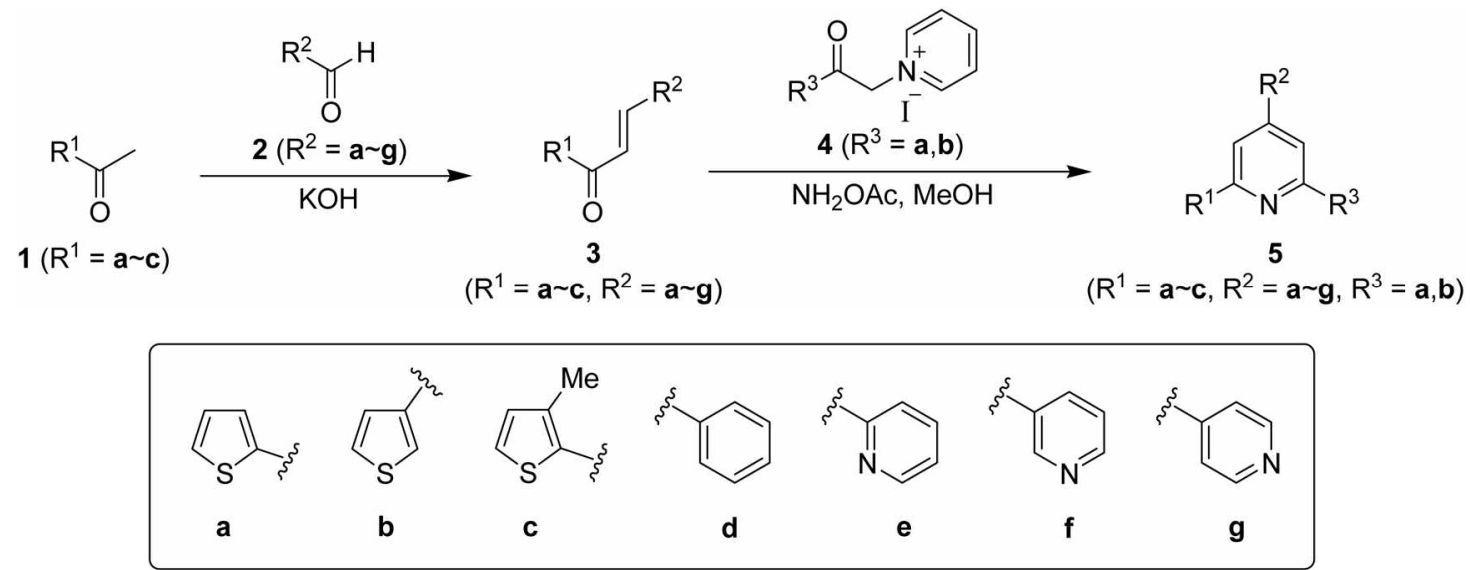

Scheme 1 
<smiles>c1csc(-c2cc(-c3cccs3)nc(-c3cccs3)c2)c1</smiles>

6<smiles>Cc1ccsc1-c1cc(-c2cccs2)nc(-c2sccc2C)c1</smiles>

12<smiles>c1csc(-c2cc(-c3ccncc3)cc(-c3cccs3)n2)c1</smiles>

18

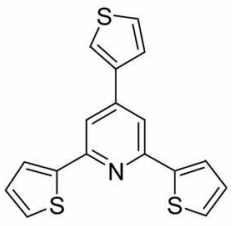

7<smiles>Cc1ccsc1-c1cc(-c2ccsc2)nc(-c2sccc2C)c1</smiles>

13<smiles>c1cncc(-c2cc(-c3ccsc3)nc(-c3cccs3)c2)c1</smiles>

19

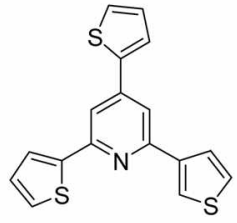

8<smiles>c1ccc(-c2cc(-c3cccs3)nc(-c3cccs3)c2)cc1</smiles>

14<smiles>c1csc(-c2cc(-c3ccncc3)cc(-c3ccsc3)n2)c1</smiles>

20<smiles>c1csc(-c2cc(-c3ccsc3)cc(-c3ccsc3)n2)c1</smiles>

9<smiles>c1ccc(-c2cc(-c3ccsc3)nc(-c3cccs3)c2)cc1</smiles>

15<smiles>c1ccc(-c2cc(-c3ccsc3)nc(-c3ccsc3)c2)nc1</smiles>

21<smiles>c1csc(-c2cc(-c3ccsc3)nc(-c3ccsc3)c2)c1</smiles>

10<smiles>c1ccc(-c2cc(-c3ccsc3)nc(-c3ccsc3)c2)cc1</smiles>

16<smiles>c1cncc(-c2cc(-c3ccsc3)nc(-c3ccsc3)c2)c1</smiles>

22<smiles>c1cc(-c2cc(-c3ccsc3)nc(-c3ccsc3)c2)cs1</smiles>

11<smiles>c1cncc(-c2cc(-c3cccs3)nc(-c3cccs3)c2)c1</smiles>

17<smiles>c1cc(-c2cc(-c3ccsc3)nc(-c3ccsc3)c2)ccn1</smiles>

23

Figure 2. The prepared compounds.

un acetate in methanol to give $6-23$ in a $54-93 \%$ yields. Pyridinium iodides ta-b were prepared in a quantitative yield by treatment of $1 \mathbf{a}-\mathbf{b}$ with iodine in pyridine. Figure 2 shows the prepared 2.6-dithienyl-4-arylpyridines (6-23)

Pharmacology. Wheat gern topoisonerase I and hunan DNA topoisomerase IIa inhibitory activities ${ }^{11}$ for the eighteen prepared 2,6-dithienyl-4-arylpyridines are shown in Figures 3 and 4 . and summarized in Table 1. Compounds 610 exhibited moderate topo I and/or II inhibitory activities.

$\begin{array}{lllllllllllllll}1 & \underline{2} & \underline{3} & \underline{4} & \underline{5} & \underline{6} & \underline{7} & \underline{8} & \underline{9} & \underline{10} & \underline{11} & \underline{12} & \underline{13} & \underline{14} & \underline{15}\end{array}$ DTC1C2 ab a bababab abab ab ab ab ab a b a b a

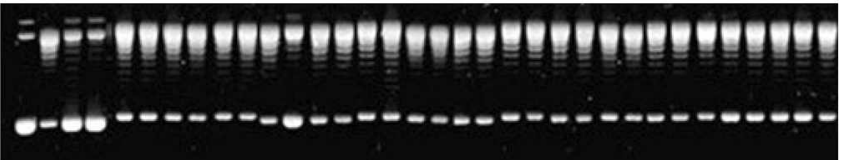

$\underline{16} \underline{17} \underline{18}$

\section{tetriget \\ $00-0$}

Lane D: pBR322 DNA only

Lane T: pBR322 DNA + Topo I

Lane C1: pBR322 DNA - Topo $1+$ Camptothecin $20 \mu \mathrm{M}$

Lane C2: pBR322 DNA - Topo I + Camptothecin $100 \mu \mathrm{M}$

Lane 1-18 Lane a: pBR322 DNA + Topo I + Compounds $20 \mu \mathrm{M}$

Lane 1-18 Lane b: pBR322 DNA - Topo I - Compounds $100 \mu \mathrm{M}$

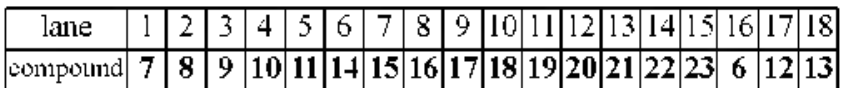

Figure 3. Topoisomerase I inhibitory activity of the prepared compounds.
Especially compound $\mathbf{6}$ indicates both moderate topo I and II inhibitory activities $(33 \%$ and $31 \%$ inhibition at $100 \mu \mathrm{M}$, respectively) and compound $\mathbf{1 0}$ has moderate topo I inhibitory activity $(35 \%$ inhibition at $100 \mu \mathrm{M})$. It is generally recognized that those compounds indicate considerable topo I or II inhibitory activities as synthetic compounds, although they have weaker inhibitory activities than those of camptothecin or etoposide. Besides the thienyl derivatives on 4-

$\begin{array}{lllllll}1 & 2 & \underline{3} & 4 & \underline{5} & \underline{6} & \underline{7}\end{array}$ $8 \quad 9 \quad 10$

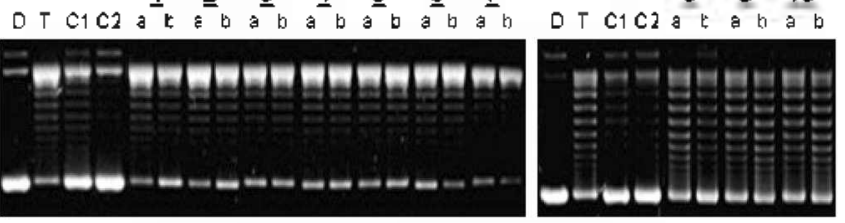

$11 \quad 12$

$\begin{array}{llllll}13 & 14 & 15 & 16 & 17 & 18\end{array}$
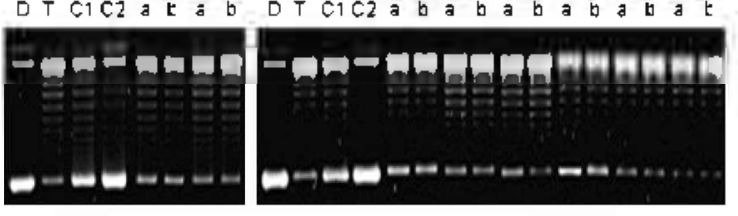

Lane D: pBR322 DNA only

Lane T: pBR322 DNA + Topo II

Lane C1: pBR322 DNA + Topo II + Etoposide $20 \mu \mathrm{M}$

Lane C2: pBR322 DNA + Topo Il + Etoposide $100 \mu \mathrm{M}$

Lane 1-18 Lane a: pBR322 DNA + Topo II + Compounds $20 \mu \mathrm{M}$

Lane 1-18 Lane b: pBR322 DNA + Topo [l + Conpounds $100 \mu \mathrm{M}$

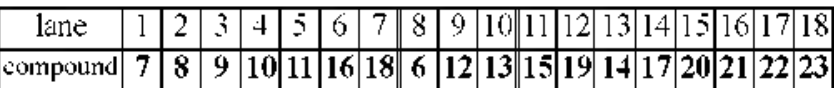

Figure 4 . Topoisomerase II inhibitory activity of the prepared compounds. 
Table 1. Topoisomerase I and II inhibitory activities of the prepared compounds

\begin{tabular}{|c|c|c|c|c|}
\hline \multirow{2}{*}{ Compounds } & \multicolumn{2}{|c|}{ Topo I (\% Inlhibition1) } & \multicolumn{2}{|c|}{ Topo II (\% Inhibitions) } \\
\hline & $20 \mu \mathrm{M}$ & $100 \mu \mathrm{M}$ & $20 \mu \mathrm{M}$ & $100 \mu \mathrm{M}$ \\
\hline 6 & 12 & 33 & 5 & 31 \\
\hline 7 & 7 & 12 & 0 & 17 \\
\hline 8 & 6 & 13 & 0 & 11 \\
\hline 9 & 10 & 6 & 5 & 2 \\
\hline 10 & 15 & 35 & 2 & 5 \\
\hline 11 & 0 & 0 & 0 & $\hat{3}$ \\
\hline 12 & 0 & 0 & 0 & 0 \\
\hline 13 & 0 & 0 & 0 & 3 \\
\hline 14 & 0 & 0 & 10 & 7 \\
\hline 15 & 0 & 0 & 12 & 15 \\
\hline 16 & 0 & 0 & 5 & () \\
\hline 17 & 0 & 0 & 0 & 0 \\
\hline 18 & 0 & 0 & 0 & 0 \\
\hline 19 & 0 & 0 & 2 & 0 \\
\hline 20 & 0 & 0 & 0 & 0 \\
\hline 21 & 0 & 7 & 13 & 7 \\
\hline 22 & 1 & 1 & 0 & 0 \\
\hline 23 & 8 & 0 & 0 & 0 \\
\hline Camptothecin & 41 & 34 & & \\
\hline Etoposide & & & 65 & 73 \\
\hline
\end{tabular}

position. other compounds 11-23 did not show considerable inhibitory activity. which indicate that phenyl or pyridyl derivatives as well as methyl thienyl on t-position in pyridine ring did not increase the inlibitory activity.

In conclusion, we have designed an efficient synthetic route to prepare eighteen 2,6-dithienyl-4-arylpyridines and evaluated them for their topo I and II inhibitory activity. From structure-activity relationship study, 2,4.6-trithienylpyridines show better inhibitory activities than 2,6-dithienyl4-phenyl or 2.6-dithienyl-4-pyridylpyridines. but lover inhibitory activities than 2,6-dithienyl-4-furylpyridines. ${ }^{\text {lo }}$ This study may provide valuable information to the researchers who are working on the development of antitumor agents. especially that of topoisomerase I or II inhibitors.

Acknowledgments. This work was supported by a grant (KRF-2006-005-J01101) from the Korea Research Foundation, R.O.K

\section{References}

1. Wang. T. C. Amm. Rev: Biochem. 1996. 65.635

2. Pommier. Y. Biochinte 1998. 80. 255.

3. Redinbo. M. R.: Stewart. L.: Kuhn1. P.: Champoux. T. T.: Hol. W. G Science 1998. 279, 504

4. Chen. A. Y.: Liu. L. F. Amu Rav Phamacol Toxicol 1994. 34, 191

5. Kellner. U.: Rudolph. P.: Parwaresch, R. Onkologie 2000, 23. 424.

6. Singh. S. K.: Ruchelman. A. L.: Li. T. K.: Liu. A.: Liu. L. F.: Lavoie. E. T. J. Med Chem. 2003. +6. 2254.

7. (a) Mukkala. V. M.: Helenius. M.: Hemmila. I.: Kankare. J.: Takalo, H. Het Chim Acta 1993. 76, 1361. (b) Mukikala. V. M: Kwiatkowski, M: Kankare, J.: Takalo. H. Helv Chim Acta 1993. 76. 893. (c) Lowe. G.; Droz, A. S.: Park, J. J.; Weaver, G. W. Bioorg. Chenistiv 1999.27.477.

8. (a) Tennette. K.: Lippard. S. T.: Vassiliades. G.: Bauer. W. Proc. Natl. Acad. Sci. YS.A. 1974. 71. 3839. (b) Liu. H. Q.: Cheung. I C.: Peng, S. M: Che C. M. J. Chem. Soc. Chem. Commm 1995 1787. (c) McCoubrey: A, Latham, H. C: Cook, P. R.: Rodger, A. Lower. G. FEBS Letters 1996, 380,73. (d) Vliet. P. M. V: Toekimin. M. S.: Haasnoot. J. G.: Reedijk. J.: Nováková. O.: Vrána. O.: Brabec. V. Inorg Chm. Acta 1995. 231.57. (e) Carter. P. J.: Cheng. C. C.: Thorp. H. H. J. Am. Chent Soc. 1998. 120. 632. (f) Lowe. G.; Droz, A. S.; Vilavan. T: Weaver. G. W: Tweedale, L.: Pratt, J. M.; Rock. P: Yardley. V.: Croft, S. L. d. Hed Chem $1999,42,999$.

9. (a) Kim. D. S. H. L.: Ashendel. C. L.: Zhou. Q.: Chang. C.-T.: Lee. E. S.: Chang. C.-J. Bicorg. Hed Chem. Lett 1998. 8. 2695. (b) Xu. W. C.: Zhou. Q:: Ashendel. C. L.: Chang. C.-T.: Chang. C.-J. Bioorg. Med Chem. Lett 1999, 9. 2279. (c) Zhao. L. X: Kim. T. S.: Ahn. S. H.; Kim. T. H.: Kim. E.-k.: Cho. W. J.: Choi. H: Lee. C. S.: Kim. J. A.: Jeong, T. C.: Chang. C.j.: Lee, E. S. Bioorg. Med. Chem. Lett 2001. H. 2659. (d) Zhao. L.-X.: Moon. Y. S.: Basnet. A.: Kim. E.-K.: Cho. W.-T.: Jahng. Y.: Park. J. G.: Jeong. T. C.: Cho. W. T.: Choi. S. U.: Lee. C. O.: Lee. S. Y.: Lee. C. S.: Lee. E.-S. Bioorg. 1fed Chem. Lett 2004, 14. 1333. (e) Zhao. L.-X.; Sherchan. J.: Park, J. K: Jahne. Y.: Jeong. B.-S.; Jeong. T. C. Lee, C. S.; Lee, E. S. Arch. Pham Res. 2006. 29. 109l. (f) Bastet. A.: Thapa. P.: Karki. R.: Na. Y.: Jahn1g. Y.: Teon1g. B.-S.: Jeong. T. C.: Lee. C. S.: Lee. E. S. Bioong Med Chent. 2007. 15.4351. (g) Son. J. K.: Zhao. L. X.: Bastet. A.: Thapa. P.: Karki, R.: Na, Y, Jahng, Y; Jeong, T. C.; Jeong, B.-S.: Lee, C. S.: Lee. E. S. Eur: J. Hed Chem. 2008. 43.675.

10. Jeong. B.-S.: Basnet, A.: Thapa, P; Karki. R; Na, Y; Jahng, Y; Jeong. T. C.: Cho. W. T.: Kwot1. Y:: Lee. C. S.: Lee. E. S subntitted for publication.

11. Fukuda. M.: Nishio. K.: Kanzawa. F.: Ogasawara. H.: Ishida. T.: Arioka, H.; Bojanowski, K: Oka, M.; Saijo, N. Cancer Res. 1996. 56.789 .

12. (a) Zecher. W.: Kröhnke. F. Chem Ber: 1961, 94, (a) 690 (b) 698. (c) 707. (b) Kröhntke. F. Angew! Chent Int Ed 1963. 2.380. (c) Kröhnke. F. Smthesis 1976. 1 . 